\title{
Breastfeeding: Expressing and supplementing
}

CPD

\section{Sara Whitburn}

\section{Background}

Breastfeeding provides positive outcomes and is recommended by the World Health Organization and Australian health guidelines. While breastfeeding is the physiologically normal way to feed an infant, there are multiple reasons that supplemental feeds (ie feeds given in addition to breastmilk) may need to be given.

\section{Objective}

The aim of this article is to outline the current evidence and resources to support general practitioners to provide advice on breastfeeding when patients are expressing and supplementing.

\section{Discussion}

There are only a few absolute and relative contraindications to breastfeeding; however, breastfeeding may be disrupted because of lactation risk factors as well as physiological, psychological and socioeconomic reasons. If breastfeeding needs to be supplemented, families can use expressed breastmilk, donor milk or formula. Supplemental devices should be chosen on the basis of cost, ease of use and access, parental preference and preferably a method that aids breastfeeding. If breastfeeding difficulties can be rectified, decreasing supplements can be considered.
BREASTFEEDING provides positive health outcomes and is recommended by the World Health Organization (WHO) ${ }^{1}$ and Australian health guidelines. ${ }^{2}$ However, results from the 2010 Australian National Infant Feeding Survey ${ }^{3}$ showed that although $96 \%$ of feeding parents initiated breastfeeding, ${ }^{3}$ only $39 \%$ of infants were still exclusively breastfed at three months of age. Approximately $40 \%$ of infants aged one month had received non-human milk (eg cow or goat) or infant formula, with the rate rising to $55 \%$ at six months of age. ${ }^{3}$

While breastfeeding is the physiologically normal way to feed an infant, there are multiple reasons that supplemental feeds (ie feeds given in addition to breastmilk) may need to be given. There are only a few absolute contraindications to breastfeeding. ${ }^{2}$ In Australia, feeding parents living with human immunodeficiency virus are advised not to breastfeed. Breastfeeding is contraindicated for patients with active tuberculosis, syphilitic lesions on the breast, active herpes lesions on the nipple, untreated brucellosis or ongoing breast cancer treatment. Metabolic conditions of the infant can also be a contraindication to breastfeeding. Relative contraindications include breastfeeding when hepatitis B positive before the infant's first hepatitis B immunisation, hepatitis $\mathrm{C}$ positive if nipples are bleeding/cracked, and using illicit drugs. ${ }^{2}$

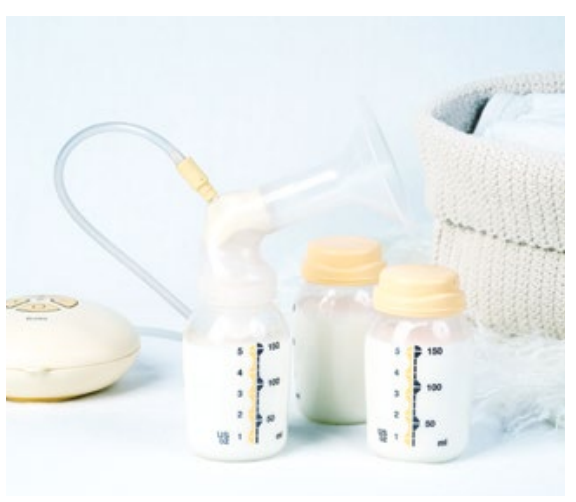

Supplements may be used because of breastfeeding disruption. This can occur as a result of lactation risk factors, ${ }^{4}$ physiological reasons, ${ }^{5}$ postnatal depression $^{6}$ and/or socioeconomic factors. ${ }^{7}$

\section{Reasons for breastfeeding disruption}

Lactation risk factors include flat or inverted nipples, history of breast surgery, low supply, attachments issues, nipple pain and previous unsuccessful breastfeeding experience. ${ }^{7}$ Physiological causes include insufficient breast development, failure of galactogenesis, maternal obesity, diabetes or infant medical or physical reasons that have an impact on feeding. Depression and anxiety can decrease oxytocin levels needed for lactogenesis as well as being independent risk factors for early discontinuation. $^{7}$

Medications are often a reason parents are advised to cease breastfeeding, but the only agents that are contraindicated include chemotherapy, radioactive pharmaceuticals, oral retinoids, amiodarone and gold salts. ${ }^{8}$ Table 1 lists further resources for advice on medication in breastfeeding.

Socioeconomic factors include parental preference, adoption, surrogacy or separation of the breastfeeding dyad, maternal educational levels and parental concern about the infant's weight gain. ${ }^{7}$ 
Socioeconomic factors can also cause unwanted lactation disruption as a result of poor access to breastfeeding support. ${ }^{7}$

General practitioners (GPs) can help to decrease breastfeeding disruption with supportive, evidence-based management of breastfeeding problems. ${ }^{9,10}$ Further information on managing common breastfeeding problems can be found using the resources listed in Table 2. For those families for whom breastfeeding is difficult or contraindicated, advice on expressing and supplementing can aid parents to achieve their breastfeeding goals.

\section{Supplementing}

Supplementing may be required for a breastfed infant if there is slow weight gain and confirmed low breastmilk supply. Before supplementing, families should have a review with a lactation consultant via lactation clinics at maternity hospitals, local councils or the Lactation Consultants of Australia and New Zealand (www. lcanz.org/find-a-lactation-consultant). Medical causes of low supply should be ruled out by checking haemoglobin level or thyroid function and ordering ultrasonography to assess retained products of conception if suspected. ${ }^{9}$
A full assessment of the infant should be performed, including investigations such as a urinary specimen (preferably clean catch) sent for microscopy and culture and any other investigations as warranted by examination.

If slow weight gain is thought to be due to the infant's inadequate intake, management involves giving the infant more calories while trying to improve the feeding parent's breastmilk supply by increasing the frequency of feeds either at the breast or through expressing.

\section{Expressing}

\section{Hand expressing}

Expressed breast milk from the infant's feeding parent is the preferred choice for infant supplementary feeding. Hand expression may achieve larger volumes than a breast pump in the first few days postpartum and can increase overall milk supply. ${ }^{5}$ The Australian Breastfeeding Association has multiple links showing videos of hand expressing that can be useful resources (www.breastfeeding.asn. au/bfinfo/hand-expressing).

\section{Breast pumps}

There are three different types of pumps: manual, electric and hospital

\section{Table 1. Resources for advice on medication in breastfeeding}

\begin{tabular}{ll} 
LactMed & www.ncbi.nlm.nih.gov/books/NBK501922 \\
\hline InfantRisk Center & www.infantrisk.com \\
\hline $\begin{array}{l}\text { The Royal Women's Hospital's Pregnancy } \\
\begin{array}{l}\text { Breastfeeding Medicine Guide (subscription } \\
\text { required) }\end{array}\end{array}$ & https://thewomenspbmg.org.au \\
\hline
\end{tabular}

\section{Table 2. Breastfeeding resources for health professionals}

The Royal Women's Hospital's breastfeeding clinical practice guidelines

Australian Breastfeeding Association Health professional webpage

Academy of Breastfeeding Medicine protocols

Lactation Consultants of Australia and New Zealand www.thewomens.org.au/healthprofessionals/maternity/breastfeedingservice

www.breastfeeding.asn.au/healthprofessionals

www.bfmed.org/protocols

www.lcanz.org/resources/medicalprofessionals/medical-provider-useful-links grade. GPs may be asked about pump recommendations. A Cochrane review analysed 22 trials involving 1339 participants to assess acceptability, effectiveness, safety, effect on milk composition and costs of methods of milk expression. ${ }^{11}$ The findings did not show a clear preference for any specific pump type. Greater milk volume was expressed when participants listened to music or used relaxation techniques, warmed and massaged the breast, pumped frequently with an appropriate breast shield size and started pumping milk frequently in the postpartum period if the infant was unable to feed at the breast. ${ }^{10}$

Breast shields are available in a range of sizes. A medium shield $(24 \mathrm{~mm})$ is the standard size usually included with breast pumps. An incorrect fit can cause nipple trauma and pain. Lubrication with purified lanolin or olive oil can be beneficial. A shield fits when the nipple moves freely and easily, without rubbing on the sides. Nipple rulers are available online to aid with fitting (https://pumpables.co/ pages/measure). Table 3 provides further sources of information on breast pumps and expressing.

Supplemental volumes should start between $30 \mathrm{~mL}$ and $60 \mathrm{~mL}$, although the amount and frequency of supplementary feeds should be aligned with infant feeding cues. ${ }^{5}$ If the volume of the feeding parent's milk does not meet the infant's requirement, donor human milk is the next option. Donor milk is available in Australia in milk banks at public hospitals and is mainly used by premature babies. The milk is screened for bloodborne viruses and pasteurised. Informal milk sharing also occurs between friends and family or arranged on social media. The Australian Breastfeeding Association's position statement on donor milk advises parents to be aware of the risks associated with privately sourced donor milk and to make informed decisions on the basis of their circumstances. ${ }^{12}$ When donor milk is not available, then formula can be offered.

\section{Infant formula feeding}

Health workers should promote breastfeeding first, but if supplemental 


\section{Table 3. Sources for information on breast pumps, expressing and storing milk}

Australian Breastfeeding Association

www.breastfeeding.asn.au or

1800 mum 2 mum (1800 686 268)

Raising Children Network

https://raisingchildren.net.au/newborns/

breastfeeding-bottle-feeding

The Royal Women's Hospital

www.thewomens.org.au/health-information/ breastfeeding

feeding is required, then health workers can educate and support parents about formula feeding. ${ }^{13}$

All infant formulas available in Australia are regulated by the Australian and New Zealand Food Standards Code and contain sufficient nutrients for infants. ${ }^{14}$ However, infant formula lacks many factors present in human milk, including cholesterol, polyamines, free amino acids, enzymes and a wide range of other bioactive substances. Bioactive agents present in breastmilk help to develop an infant's gastrointestinal tract, immune system and brain, and they provide protection against later metabolic disease. $^{15}$

There is minimal evidence that if breastfeeding is disrupted, any specific formula is superior for healthy full-term infants without allergies. ${ }^{3}$ Infants with allergies can use extensively hydrolysed, rice or amino acid-based formulas. A paediatric review is recommended for assessment and prescription for most of these formulas. Soy formula, if tolerated, can be used from six months of age.

The use of 'follow-on formula' for infants aged 6-12 months is not considered necessary and has not been shown to have advantages over using 'infant formula'. Further information about formula feeding - including preparation, handling and storage can be found on the Raising Children Network website (https://raisingchildren. net.au/newborns/breastfeeding-bottlefeeding/bottle-feeding/infant-formula).

\section{Supplemental devices}

When supplemental feeding is required, several delivery methods are available.

These include supplemental nursing devices at the breast (SNS), cup feeding, spoon feeding, syringe feeding or bottle feeding.

When choosing an alternative feeding method, criteria that should be considered include:

- cost and availability

- practicality and cleaning needs

- whether anticipated use is short- or long-term

- parental preference

- the expertise of supporting health practitioners

- whether the method aids the development of breastfeeding skills.

Cup feeding allows infants to control feeding pace. ${ }^{5}$ Cup feeding has been shown to be safe for both term and preterm infants. ${ }^{5}$

SNS supply supplementation enables and encourages the feeding parent, while simultaneously stimulating the breast to produce more milk, maintaining the infant's feeding at the breast, enabling the feeding parent to have a breastfeeding experience and encouraging skin-to-skin contact. However, the system can be awkward to use, difficult to clean and expensive; it requires parental upskilling, and the infant must be able to latch effectively. ${ }^{5}$

Bottle feeding is the most used method of supplementation. However, there are some concerns about infant safety when using bottles. ${ }^{16}$ Infants can have difficulties coordinating swallowing and respiration because of distinct differences in tongue and jaw movements when sucking on a teat. Faster flow may result in higher volumes of feeds, possibly leading to overfilling of the mouth and resulting in choking. ${ }^{5,16}$ Preterm infants are at greater risk of choking than term infants because of immature or disorganised sucking patterns, poor regulation of respiration and an immature gastrointestinal tract. ${ }^{17}$
Some experts have recommended a teat/nipple with a wide base and slow flow to try to mimic breastfeeding and to avoid nipple preference, ${ }^{18}$ but little research has been done evaluating outcomes with different teats/nipples. ${ }^{5}$ If bottles are being used, pacing the feed supports safe sucking and respiration patterns. ${ }^{19}$ Possums \& Co. has an online video explaining paced feeding (https:// possumsonline.com/video/about-pacedbottle-feeding-renee-keogh).

\section{Transitioning from supplementing}

If the breastfeeding difficulties resolve or infant weight gains improve, discussion of transitioning from supplements can occur. This should be done with the support of a lactation consultant, maternal child health nurse and medical team. When weaning off formula supplements, the aim is to increase the infant's breastmilk intake while decreasing the volume and frequency of formula supplements.

A small amount (eg $30 \mathrm{~mL}$ ) of formula may be reduced over 24 hours and replaced with more frequent breastfeeds or expressed breastmilk (aiming for 8-12 breastfeeds in 24 hours). Weekly weighing and careful monitoring of the infant's output and hydrations status are needed.

Table 2 lists further breastfeeding resources for health professionals.

\section{Conclusion}

There are very few reasons that breastfeeding is absolutely contraindicated; however, as a result of lactation risk factors as well as physiological, psychological and socioeconomic factors, breastfeeding can be disrupted. Breastfeeding may need to be supplemented by expressed breastmilk, donor milk or formula. GPs can use resources to provide information on expressing, breast pumps and formula preparation. Supplemental devices should be chosen on the basis of cost, ease of use and access, parental preference and preferably a method that supports breastfeeding. If breastfeeding difficulties can be rectified, decreasing supplements can be considered. 


\section{Author}

Sara Whitburn FRACGP, DRANZCOG, IBCLE DFSRH, Medical Educator, Sexual Health Victoria, Box Hill, Vic

Competing interests: None.

Funding: None.

Provenance and peer review: Commissioned, externally peer reviewed.

\section{Correspondence to:}

swhitburn@shvic.org.au

\section{Acknowledgements}

Dr Whitburn would like to acknowledge Dr Lisa Amir and Dr Anita Bearzatto for advice on the draft of this article.

\section{References}

1. World Health Organization. Exclusive breastfeeding for six months best for babies everywhere. Geneva, $\mathrm{CH}$ : WHO 2011. Available at www.who.int/news/ item/15-01-2011-exclusive-breastfeeding-for-sixmonths-best-for-babies-everywhere [Accessed 23 May 2021].

2. National Health and Medical Research Council. Infant feeding guidelines: Summary. Canberra, ACT: NHMRC, 2013.

3. Australian Institute of Health and Welfare. 2010 Australian national infant feeding survey: Indicator results. Cat. no. PHE 156. Canberra, ACT: AlHW, 2011.

4. Vanguri S, Rogers-McQuade H, Sriraman NK Academy of Breastfeeding Medicine. ABM clinica protocol \#14: Breastfeeding-friendly physician's office-optimizing care for infants and children. Breastfeed Med 2021;16(3):175-84. doi: 10.1089/ bfm.2021.29175.sjv.
5. Kellams A, Harrel C, Omage S, Gregory C, Rosen-Carole C. ABM clinical protocol \#3: Supplementary feedings in the healthy term breastfed neonate, revised 2017. Breastfeed Med 2017;12:188-98. doi: 10.1089/bfm.2017.29038.ajk

6. Taveras EM, Capra AM, Braveman PA, Jensvold NG, Escobar GJ, Lieu TA. Clinician support and psychosocial risk factors associated with breastfeeding discontinuation. Pediatrics 2003;112(1 Pt 1):108-15. doi: 10.1542/peds.112.1.108.

7. Stuebe AM, Horton BJ, Chetwynd E, Watkins S, Grewen K, Meltzer-Brody S. Prevalence and risk factors for early, undesired weaning attributed to lactation dysfunction. J Womens Health (Larchmt) 2014;23(5):404-12. doi: 10.1089/jwh.2013.4506.

8. Sachs $\mathrm{HC}$; Committee on Drugs. The transfer of drugs and therapeutics into human breast milk: An update on selected topics. Pediatrics 2013;132(3):e796-809. doi: 10.1542/peds.20131985.

9. Amir LH. Breastfeeding - Managing 'supply' difficulties. Aust Fam Physician 2006;35(9):686-89.

10. Amir LH. Managing common breastfeeding problems in the community. BMJ 2014;348:g2954. doi: 10.1136/bmj.g2954.

11. Becker GE, Smith HA, Cooney F. Methods of milk expression for lactating women. Cochrane Database Syst Rev 2016;9(9):CD006170. doi: 10.1002/14651858.CD006170.pub5.

12. Australian Breastfeeding Association. Position statement on donor milk. South Melbourne, Vic: ABA, 2014.

13. World Health Organization. The international code of marketing of breast-milk substitutes. Geneva, $\mathrm{CH}$ : WHO, 1981

14. Food Standards Australia New Zealand. Australia New Zealand food standards code. Canberra, ACT: FSANZ, 2019.
15. Martin CR, Ling PR, Blackburn GL. Review of infant feeding: Key features of breast milk and infant formula. Nutrients 2016;8(5):279. doi: 10.3390/nu8050279.

16. Pados BF, Park J, Thoyre SM, Estrem H, Nix WB Milk flow rates from bottle nipples used for feeding infants who are hospitalized. Am J Speech Lang Pathol 2015;24(4):671-79. doi: 10.1044/2015 AJSLP-15-0011.

17. Nyqvist $\mathrm{KH}$. Early attainment of breastfeeding competence in very preterm infants. Acta Paediatr 2008;97(6):776-81. doi: 10.1111/j.16512227.2008.00810.x.

18. Kassing D. Bottle-feeding as a tool to reinforce breastfeeding. J Hum Lact 2002;18(1):56-60. doi: 10.1177/089033440201800110.

19. Law-Morstatt L, Judd DM, Snyder P, Baier RJ, Dhanireddy R. Pacing as a treatment technique for transitional sucking patterns. J Perinatol 2003;23(6):483-88. doi: 10.1038/sj.jp.7210976.

correspondence ajgp@racgp.org.au 